OPEN ACCESS

Edited by:

Jian-Yong Li,

Nanjing Medical University, China

Reviewed by:

Mingzhi Zhang,

First Affiliated Hospital of Zhengzhou

University, China

Lei Fan,

The First Affiliated Hospital of Nanjing

Medical University, China

${ }^{*}$ Correspondence:

Wei Zhang

vv1223@vip.sina.com

Specialty section:

This article was submitted to

Pharmacology of Anti-Cancer Drugs,

a section of the journal

Frontiers in Oncology

Received: 18 August 2021 Accepted: 09 December 2021 Published: 24 December 2021

Citation:

Zhang Y, Li Y, Zhuang Z, Wang W, Wei $C$, Zhao $D$, Zhou $D$ and Zhang $W$ (2021) Preliminary Evaluation of Zanubrutinib-Containing

Regimens in DLBCL and the

Cerebrospinal Fluid Distribution of Zanubrutinib: A 13-Case Series.

Front. Oncol. 11:760405.

doi: 10.3389/fonc.2021.760405

\section{Preliminary Evaluation of} Zanubrutinib-Containing Regimens in DLBCL and the Cerebrospinal Fluid Distribution of
Zanubrutinib: A 13-Case Series

\author{
Yan Zhang, Yanan Li, Zhe Zhuang, Wei Wang, Chong Wei, Danqing Zhao, \\ Daobin Zhou and Wei Zhang*
}

Department of Hematology, Peking Union Medical College Hospital, Chinese Academy of Medical Sciences (CAMS) \& Peking Union Medical College, Beijing, China

Zanubrutinib is a second-generation Bruton's tyrosine kinase inhibitor. Its safety and effectiveness in central nervous system (CNS) lymphoma along with its distribution in the brain and ability to cross the blood-brain barrier (BBB) remain unknown. This retrospective case series involved patients with diffuse large B-cell lymphoma (DLBCL) treated with zanubrutinib-containing regimens from August to December 2020 in $\mathrm{PUMCH}$. The amounts of zanubrutinib in the plasma and brain were assessed by liquid chromatography-tandem mass spectrometry in paired plasma and cerebrospinal fluid (CSF) samples. In total, 13 patients were included: eight primary CNS lymphoma cases and five systemic DLBCL cases with 61.5\% (8/13) refractory/relapsed and 84.6\% (11/13) showing CNS involvement. The overall response rates were $84.5 \%$ in the entire population and $81.8 \%$ in the CNS-involved cases. A total of 23 time-matched plasma-CSF sample pairs were collected. The mean peak concentration of zanubrutinib in CSF was 2941.1 $\mathrm{pg} / \mathrm{ml}$ (range, 466-9032.0 pg/ml). The corrected mean CSF/plasma ratio determined based on 94\% protein binding was $42.7 \% \pm 27.7 \%$ (range, $8.6 \%-106.3 \%$ ). This preliminary study revealed the effectiveness of zanubrutinib-containing regimens in DLBLC, especially CNS-involved cases, for the first time. The excellent BBB penetration of zanubrutinib supports its further investigation for the treatment of CNS lymphoma.

Keywords: Zanubrutinib, diffuse large B-cell lymphoma, liquid chromatography-tandem mass spectrometry, cerebrospinal fluid, distribution 


\section{INTRODUCTION}

B-cell receptor (BCR) signaling is essential for normal B-cell proliferation, differentiation, survival, and function. Aberrant BCR signaling pathway activation is consequently observed in various B-cell malignancies (1). Therefore, blocking BCR signaling is an attractive strategy for therapeutic intervention in B-cell malignancies. Bruton's tyrosine kinase (BTK) is a key effector of BCR signaling. BTK inhibitors including ibrutinib, zanubrutinib, and acalabrutinib show significant clinical effects in chronic lymphocytic leukemia (CLL), Waldenström macroglobulinemia (WM), and mantle cell lymphoma (MCL). The first-generation BTK inhibitor ibrutinib shows significant antitumor activity in relapsed/refractory activated B cell-like (ABC) diffuse large B-cell lymphoma (DLBCL) (2). Very recently, after analysis of biopsies from Phoenix trial, Wilson. et al. reported that the 3-year event-free survival of younger patients treated with ibrutinib plus R-CHOP was $100 \%$ in the MCD subtypes, which provide solid evidence of the efficacy of ibrutinib in DLBCL (3) .

Central nervous system lymphoma (CNSL) remains a great clinical challenge; relapse eventually occurs in over $60 \%$ of primary CNSL cases, and the median overall survival period is less than 12 months for secondary CNSL $(4,5)$. The unmet need for effective biological therapies stems from the impermeability of the bloodbrain barrier (BBB). The BBB restricts entry to the brain due to its very low paracellular permeability. Only lipophilic drugs can easily cross the BBB by passive diffusion, while some can permeate the $\mathrm{BBB}$ via potent influx transporters (6). Drug efficacy in CNSL requires the drug to pass through the $\mathrm{BBB}$ and distribute in the brain. Methorexate (MTX) based protocols are widely used, such as MA (methotrexate, cytarabine), MATRiX (MTX, cytarabine, thiotepa, rituximab), MVP (MTX, vincristine, procarbazine), however, the complete remission rate was less than $60 \%(7,8)$. Adding small molecular drugs with good penetration of brain blood-barrier (BBB) may induce higher response rate, such as lenalidomide (NCT04120350). Secondly, the optimal prophylaxis strategy is undetermined. Intrathecal or intravenous MTX has been used for a longtime, but the efficacy was disappointed, the CNS relapsing rate was $>10 \%$ in the high risk group after high-dose MTX $(9,10)$. In view of the above-mentioned facts, we need a novel medication with good BBB penetration to overcome difficulties in CNLS treatment.

BTK inhibitors may be effective for primary central nervous system lymphoma (PCNSL). Various case reports have demonstrated that ibrutinib crosses the $\mathrm{BBB}$, leading to complete remission (11-14). Analytical methods have been reported for quantifying the active metabolites of ibrutinib in animal models along with human plasma and cerebrospinal fluid (CSF) (15-18). Tirabrutinib, another BTK inhibitor, has also shown good efficacy in patients with relapsed/refractory PCNSL and was approved on March 25, 2020 in Japan (19).

Zanubrutinib (BRUKINSA ${ }^{\mathrm{TM}}$, Beigene Corporation; also known as BGB-3111) is an irreversible next-generation BTK inhibitor that was approved for the treatment of recurrent or refractory CLL, MCL, and WM (20-22). Zanubrutinib was designed with favorable pharmacokinetic and pharmacodynamic (PD) features to maximize BTK occupancy and minimize off-target inhibition (23).

However, there is no clinical evidence of zanubrutinib in DLBCL with CNS involvement. Therefore, we reviewed the clinical profiles of several aggressive B-cell lymphoma cases treated with zanubrutinib to investigate the effectiveness and safety of zanubrutinib. We hypothesized that zanubrutinib effectively permeates the $\mathrm{BBB}$ and is effective for the treatment of primary and secondary CNSL. To the best of our knowledge, no method has been specifically developed for the quantification of zanubrutinib in the CSF. Thus, we developed a sensitive and rapid liquid chromatography-tandem mass spectrometry (LC-MS/MS) method for the quantification of zanubrutinib in plasma and CSF, evaluated the distribution of zanubrutinib in CSF, and preliminarily assessed the safety and effectiveness of zanubrutinib against DLBCL.

\section{MATERIALS AND METHODS}

\subsection{Patients}

Patients with treatment-naive and relapsed/refractory DLBCL were enrolled in this study. The inclusion criteria were as follows: age > 18 years; histopathologically confirmed DLBCL (including DLBCL-NOS, PCNSL, and IVLBCL); assessable lesions by computed tomography or magnetic resonance imaging; and treatment with zanubrutinib-containing regimens. The exclusion criteria were as follows: severe disease complications or serious comorbidities; active infection; uncontrolled bleeding events; and pregnancy or lactation in women.

This study was conducted in accordance with the ethics principles of the Declaration of Helsinki, and the study methods were approved by the Institutional Review Board of Peking Union Medical College Hospital. Informed consent was obtained from all patients.

\subsection{Treatment}

The dose of zanubrutinib was fixed at $160 \mathrm{mg}$ twice per day in all patients. Treatment duration and the combined drugs/regimens were decided by the clinicians.

Four regimens were evaluated in this study: Regimen 1, zanubrutinib + rituximab in primary vitreoretinal lymphoma (PVRL) (zanubrutinib at $160 \mathrm{mg}$ twice per day until disease progression or intolerance and rituximab at $375 \mathrm{mg} / \mathrm{m}^{2}$ every 21 days); Regimen 2, zanubrutinib combined with high-dose methotrexate with or without cytarabine (HD-MTX regimen/ MA regimen) in CNSL (methotrexate at $3.5-5.0 \mathrm{~g} / \mathrm{m}^{2} \mathrm{~d} 1$ and cytarabine at $2.0 \mathrm{~g}$ every $12 \mathrm{~h}$ on $\mathrm{d} 2$ and $\mathrm{d} 3$, every 21 days per cycle); Regimen 3, zanubrutinib combined with dexamethasone, ifosfamide, carboplatin, and etoposide (DICE) or dexamethasone, cytarabine, and cis-platinum (DHAP) in relapsed and refractory patients every 21 days per cycle as prescribed for the salvage chemotherapy of autologous stem cell transplantation (ASCT); and Regimen 4, zanubrutinib combined with rituximab, cyclophosphamide, epirubicin, vindesine, and prednisone (R-CHOP) for eight cycles as an initial treatment in IVLBCL. 


\subsection{Quantification of Zanubrutinib in Peripheral Blood and CSF Samples}

\subsubsection{Plasma and CSF Sample Preparations}

Zanubrutinib is absorbed rapidly with a median time to maximum concentration of $2 \mathrm{~h}$. Blood and CSF samples were collected at 2 or $3 \mathrm{~h}$ after the oral administration of zanubrutinib at $160 \mathrm{mg}$. Zanubrutinib administration was performed for more than $7 \mathrm{~d}$. Additional plasma and CSF specimens for trough concentration testing were collected just before zanubrutinib administration (i.e., $12 \mathrm{~h}$ after the last zanubrutinib dose). Biological samples were stored at $-20^{\circ} \mathrm{C}$ for a maximum of one month before analysis.

\subsubsection{Materials, Instruments, and Procedures}

Zanubrutinib in CSF and sodium heparin plasma samples was quantified by LC-MS/MS. Zanubrutinib standard was obtained from Beigene (Beijing, China). Methanol, methanoic acid, and acetonitrile (high-performance liquid chromatography gradient grade) were purchased from Fisher Scientific, USA. Ultrapure water was obtained using a Milli-Q purification system (Millipore, MA, USA). Control plasma and CSF samples for standards and quality control were obtained from patients treated in the department of hematology of PUMCH. The detailed LC-MS/MS procedure is described in the Supplementary Material.

\subsection{Clinical Outcomes and Adverse Events (AEs)}

The clinical response was assessed based on the Lugano 2014 Criteria for DBLCL and 2005 IELSG Criteria for PCNSL $(24,25)$.
AEs were recorded and assessed according to the Common Terminology Criteria for Adverse Events (CTCAE) v4.0.

\subsection{Statistical Analysis}

Continuous variables were expressed as median and range and compared by Mann-Whitney U-test. Categorical variables were reported as percentages and compared by chi-squared test. The duration of response (DOR) was determined using the KaplanMeier method. Statistical analyses were performed with SPSS 25.0 (IBM, NY, USA) or Prism 7.0 (GraphPad, CA, USA). $P<0.05$ was considered statistically significant.

\section{RESULTS}

\subsection{Clinical Characteristics and Treatment}

A total of 13 patients were included from August to December 2020. The median age was 53 (range, 39-69) years, and four patients were male. The histological subtypes were PCNSL $(n=8)$, DLBCL-NOS $(n=2)$, PTL $(n=1)$, and IVLBCL $(n=2)$; all eight PCNSL cases were PVRL with or without brain involvement. The median IELSG score of the PCNSL cases was $0(0-1)$. The median IPI (International Prognosis Index) score of the five systemic DLBCL cases was $4(0-4)$, and four out of those five cases were Ann Arbor IV stage. A total of seven cases were relapsed, with a median of two (range, 1-3) prior treatments; six patients were newly diagnosed with primary vitreoretinal lymphoma and IVLBCL. A total of seven patients had CNS involvement; of these, four cases showed PRVL relapse with intracerebral lesions, and three had DLBCL with systemic and

\begin{tabular}{|c|c|c|c|c|c|c|c|c|c|c|}
\hline ID & Age & Sex & Diagnosis & Comorbidity & Status & $\begin{array}{l}\text { Lesion location } \\
\text { @diagnosis }\end{array}$ & Previous treatments & $\begin{array}{l}\text { Lesion location } \\
\text { @relapsing }\end{array}$ & Regimen & $\begin{array}{c}\text { Best } \\
\text { response/ } \\
\text { DOR }\end{array}$ \\
\hline 1 & 69 & $M$ & PCNSL & HTN & $\mathrm{TN}$ & eyes & NA & NA & $\mathrm{R}+$ Zan & $\mathrm{CR} / 11 \mathrm{~m}$ \\
\hline 2 & 53 & $\mathrm{~F}$ & PCNSL & Ovarian tumor & $\mathrm{TN}$ & eyes & NA & NA & $\mathrm{R}+$ Zan & $\mathrm{CR} / 9 \mathrm{~m}$ \\
\hline 3 & 44 & $\mathrm{~F}$ & PCNSL & $\mathrm{HCV}$ & $\mathrm{TN}$ & eyes & NA & NA & $\mathrm{R}+$ Zan & $\mathrm{CR} / 9 \mathrm{~m}$ \\
\hline 4 & 52 & $\mathrm{~F}$ & PCNSL & & TN & eyes & NA & NA & $\mathrm{R}+$ Zan & $\mathrm{CR} / 9 \mathrm{~m}$ \\
\hline 5 & 50 & $\mathrm{~F}$ & PCNSL & $\begin{array}{l}\text { breast cancer, } \\
\text { paraganglioma }\end{array}$ & relapsed & eyes & $\begin{array}{l}\text { rituximab/Lenalidomide } 6 \text {, } \\
\mathrm{R}-\mathrm{MTX} \times 2, \text { etoposide } \times 2\end{array}$ & $\begin{array}{l}\text { left frontal lobe, } \\
\text { cerebellum }\end{array}$ & Zan+MA & $\mathrm{CR} / 14 \mathrm{~m}$ \\
\hline 6 & 62 & $\mathrm{~F}$ & PCNSL & hypertension & relapsed & eyes & rituximab/lenalidomide×6 & right frontal lobe & Zan+R+HD-MTX & $\mathrm{CR} / 13 \mathrm{~m}$ \\
\hline 7 & 59 & $\mathrm{~F}$ & PCNSL & $\mathrm{HBV}$ & relapsed & eyes & Rituximab+lenalidomide×6, & $\begin{array}{l}\text { bilateral basal } \\
\text { ganglia }\end{array}$ & Zan+HD-MTX & SD/NA \\
\hline 8 & 52 & $\mathrm{~F}$ & PCNSL & & relapsed & eyes & rituximab/Lenalidomide×6, & $\begin{array}{l}\text { bilateral frontal } \\
\text { lobes, callosum }\end{array}$ & Zan+R+HD-MTX & $\mathrm{CR} / 13 \mathrm{~m}$ \\
\hline 9 & 63 & $M$ & PTL & & refractory & testis, epididymis & R-EPOCH/R-MA & testis, epididymis & Zan+R-DICE & $\mathrm{CR} / 11 \mathrm{~m}$ \\
\hline 10 & 39 & $\mathrm{~F}$ & $\begin{array}{l}\text { DLBCL, } \\
\text { NOS }\end{array}$ & HBV & relapsed & $\begin{array}{l}\text { bone marrow, lung, } \\
\text { uterus }\end{array}$ & $\begin{array}{l}\text { R-MTX-CHOP, ASCT, } \\
\text { TEDDI-R }\end{array}$ & right frontal lobe & Zan+MA & PD/NA \\
\hline 11 & 60 & $M$ & $\begin{array}{l}\text { DLBCL, } \\
\text { NOS }\end{array}$ & & relapsed & $\begin{array}{l}\text { cervical lymph } \\
\text { nodes }\end{array}$ & & $\begin{array}{l}\text { muscles of right } \\
\text { thigh }\end{array}$ & Zan+R-DHAP & $\mathrm{CR} / 13 \mathrm{~m}$ \\
\hline 12 & 65 & M & IVBCL & diabetes mellitus & TN & $\begin{array}{l}\text { lung, bone marrow, } \\
\text { liver }\end{array}$ & NA & NA & Zan+R-CHOP & $\mathrm{CR} / 12 \mathrm{~m}$ \\
\hline 13 & 53 & $\mathrm{~F}$ & IVBCL & hypothyroidism & $\mathrm{TN}$ & CNS, skin, lung & R-CHOP-MTX×8 & NA & Zan & $\mathrm{PR} / 13 \mathrm{~m}$ \\
\hline
\end{tabular}

COO, cell of origin; CNS, central nerves system; CR, complete remission; CHOP, cyclophosphamide, epirubicin, vindesine, prednisone; DICE, dexamethasone, ifosfamide, carboplatin and etoposide; DHAP, dexamethasone, cytarabine and cis-platinum; DOR, duration of response; EPOCH, etoposide, prednisone, vindesine, cyclophosphamide, epirubicin; $F$, female; GCB, germinal center B cell; HD, high dose; HBV, hepatitis B virus; HCV, hepatitis C virus; IVLBCL, intravascular large B cell lymphoma; MA, methotrexate, cytarabine; M, male; MTX, methotrexate, MT, maintenance; PCNSL, primary central nervous system lymphoma; PD, progressed disease; PR, partial remission; PTL, primary testis lymphoma; $R$, rituximab; TN, treat naive; NA, not applicable; Zan, zanubrutinib. 
intracranial lesions. The detailed clinical and histopathological characteristics are shown in Table 1.

A total of four PVRL patients without CNS involvement were treated with zanubrutinib and rituximab as a first-line treatment. Zanubrutinib and high-dose (HD) methotrexate (MTX)-based regimens were applied in five patients with isolated CNS involvement; these regimens included MTX combined with rituximab and/or cytarabine (HD-MTX, $n=1$; R-HD-MTX, $\mathrm{n}=2$; R-MA, $n=2$ ). Two patients with relapsed/refractory DLBCL received rituximab and zanubrutinib combined with DICE or DHAP as salvage regimens. Two patients with IVLBCL had systemic CNS involvement at diagnosis; one was treated with zanubrutinib combined with $\mathrm{R}-\mathrm{CHOP}$ as a first-line therapy, while the other received R-CHOP-MTX for eight cycles and achieved partial response (PR). The patient achieving PR was further treated with zanubrutinib as a maintenance therapy (Figure 1).

\subsection{Clinical Outcomes}

All five treatment-naïve patients (one IVBLC and four PRVL patients) achieved complete response (CR) after six to eight cycles of zanubrutinib-containing first-line regimens. The two relapsed/refractory DLBCL cases without CNSL involvement achieved CR and received ASCT as consolidation.

The response rate for the five relapsed CNSL patients was $60 \%(3 / 5)$, including three patients with CR, one with stable disease (SD), and one with disease progression. Of these five patients, two first received zanubrutinib monotherapy for over seven days as a lead-in therapy before combination chemotherapy, and a rapid and dramatic response was observed in both of these patients. Case 5 was a 50 -year-old woman diagnosed with PRVL who relapsed in bilateral eyes and the frontal lobe in sequence after first-line treatment with rituximab and lenalidomide. Short durations of complete remission (four and three months, respectively) were achieved after the administration of rituximab combined with HD MTX and $\mathrm{HD}$ etoposide as salvage treatments. At the third relapse, she was diagnosed with bladder paraganglioma that required surgery. Zanubrutinib was administered only around the operative period; consequently, the lesion in the cerebellum shrank and disappeared within three weeks (Figure 2). Case 6 was a 62-year-old female with PRVL who was treated with rituximab and lenalidomide as a frontline treatment followed by lenalidomide as a maintenance therapy. Although the patient achieved CR according to IELSG Criteria 2005, the amount of IL10 in the CSF remained around $400 \mathrm{ng} / \mathrm{ml}$ (normal range, $<5 \mathrm{ng}$ / $\mathrm{ml})$. The CSF IL-10 concentration is strongly associated with the clinical response and/or progression $(26,27)$. After the disease relapsed 18 months later, the patient underwent zanubrutinib monotherapy for $7 \mathrm{~d}$ before systemic intravenous MTX administration. The CSF IL-10 concentration was dramatically decreased by the zanubrutinib monotherapy, which was related to the control of CNS lymphoma and demonstrates the significant anti-tumor activity of zanubrutinib (Figure 3).

The overall response rate (ORR) of the entire population was $84.5 \%(11 / 13)$, and the CR rate was $76.9 \%(10 / 13)$. After a median follow-up of 3.6 (6-14) months, the DOR was 12 (914) months. Only one patient died from disease progression.

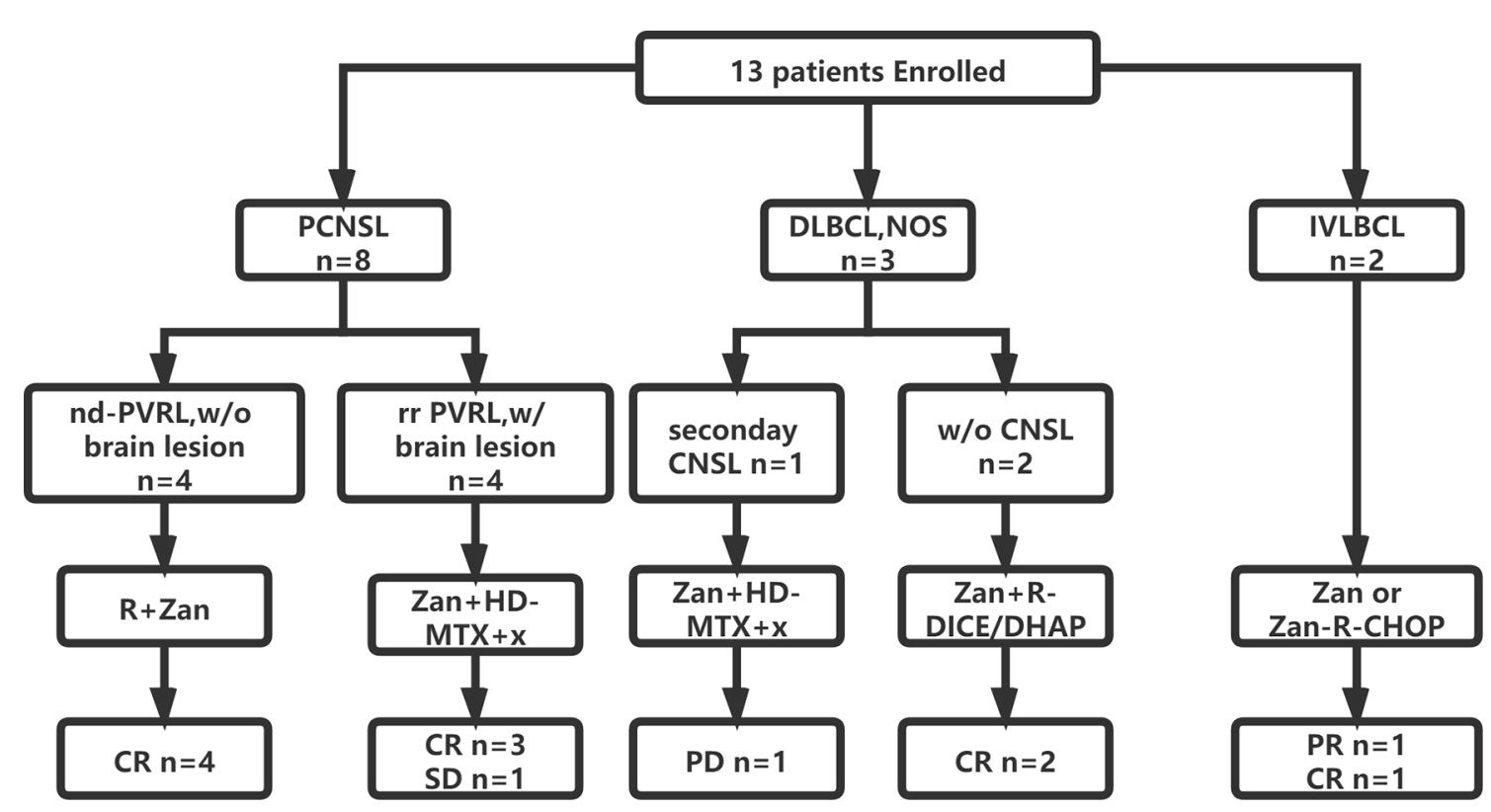

FIGURE 1 | Flowchart of patient enrollment, treatment and outcome. CNS, central nerves system; CR, complete remission; CHOP, cyclophosphamide, epirubicin, vindesine, prednisone; DICE, dexamethasone, ifosfamide, carboplatin and etoposide; DHAP, dexamethasone, cytarabine and cis-platinum; HD, high dose; IVLBCL, intravascular large B cell lymphoma; MTX, methotrexate; nd, newly-diagnosed; R, rituximab; PCNSL, primary central nervous system lymphoma; PVRL, primary vitreoretinal lymphoma; PR, partial remission; PD, progressed disease; w/, with; w/o, without; Zan, zanubrutinib. 

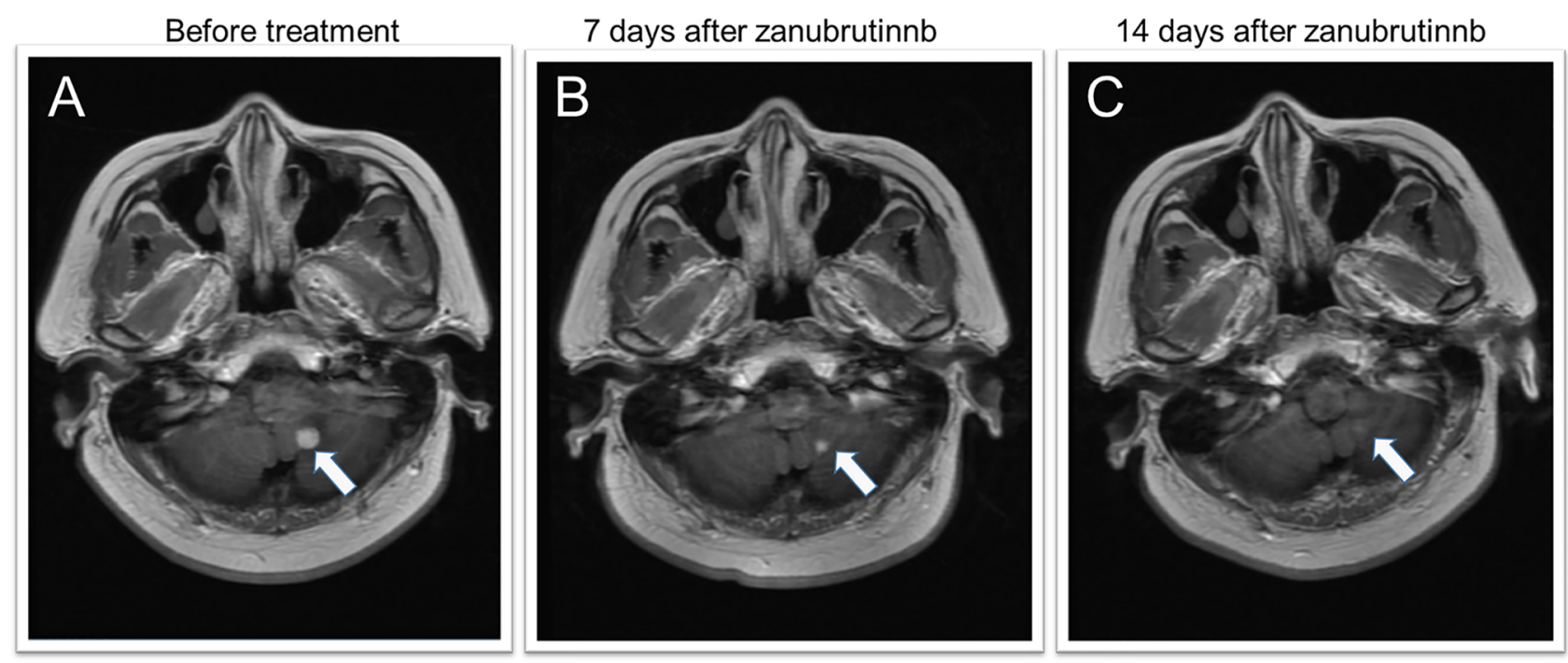

FIGURE 2 | MRI features of a secondary CNSL case during zanubrutinib monotherapy. (A) MRI depicts a homogeneously contrast-enhanced mass in the left cerebellum before zanubrutinib administration. (B, C) 7 and 14 days after zanubrutinib, the mass shrank and disappeared.

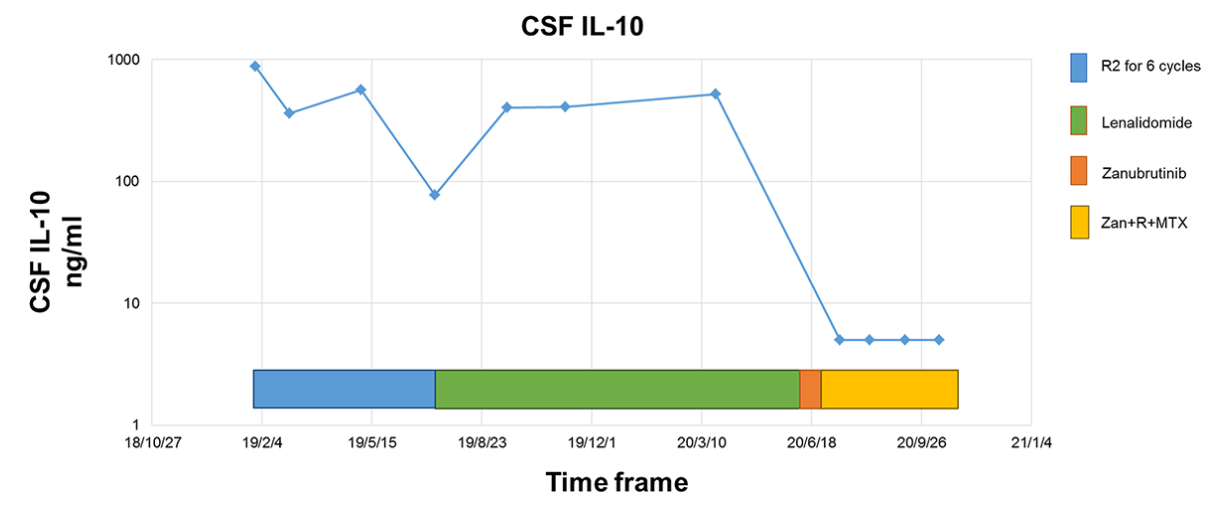

FIGURE 3 | Dynamic change of CSF IL-10 concentrations during the whole treatment.

Due to the heterogeneity of the zanubrutinib-based regimens, the categories and severity of AEs varied greatly among the different regimens. The median treatment cycle number was four (range, 2-8). The treatment was discontinued early because of disease progression in only two cases, and the remaining patients completed the planned cycles $(n=4)$ or were still under treatment $(n=7)$. The most common AEs $(>10 \%)$ were neutropenia, thrombocytopenia, infection, nausea, vomiting, abnormal alanine transaminase, and mucositis; no bleeding or cardiac events occurred during treatment (Table 2).

\subsection{CSF Distribution of Zanubrutinib}

A total of 23 time-matched plasma-CSF sample pairs were collected from the 13 patients. Due to the rapid absorption and distribution of zanubrutinib, the time to maximum concentration is approximately $2 \mathrm{~h}$. Therefore, the sampling time was selected as $2-3 \mathrm{~h}$ after zanubrutinib administration.

The mean plasma concentration of zanubrutinib was $143190.6 \pm 93302.7 \mathrm{pg} / \mathrm{ml}$ (range, 9281.0-369380.0 pg/ml) at the peak time point median CSF concentration was $2941.1 \pm$ $2382.01 \mathrm{pg} / \mathrm{ml}$ (range, 466-9032.0 pg/ml) (Figure 4). The median CSF/plasma ratio was $2.39 \% \pm 1.71 \%$ (range, $0.61 \%$ $5.83 \%$ ), indicating a low $\mathrm{BBB}$ penetration for zanubrutinib. However, considering the high protein binding rate of $94 \%$, the corrected CSF/plasma ratio was $42.7 \% \pm 27.7 \%$ (range, $8.6 \%$ $106.3 \%)$. The increase in this corrected CSF/plasma ratio demonstrates that free zanubrutinib was successfully transported from the blood into the CSF. The peak CSF 
TABLE 2 | Adverse reactions of zanubrutinib-containing regimens.

\begin{tabular}{|c|c|c|c|c|c|c|c|}
\hline $\mathrm{AE}$ & Severity & $\begin{array}{l}\text { Zan+R } \\
(n=5)\end{array}$ & $\begin{array}{c}\mathrm{Zan}+\mathrm{MTX} \pm \mathrm{R} \\
(n=3)\end{array}$ & $\begin{array}{c}\text { Zan+MA } \\
(n=2)\end{array}$ & $\begin{array}{c}\mathrm{Zan}+\mathrm{CHOP} \\
(n=1)\end{array}$ & $\begin{array}{c}\text { Zan+DICE/DHAP } \\
(n=2)\end{array}$ & $\begin{array}{l}\text { Total } \\
(n=13)\end{array}$ \\
\hline neutropenia & any grade & 1 & 3 & 2 & 1 & 2 & 9 \\
\hline \multirow[t]{2}{*}{ Thrombocytopenia } & any grade & 0 & 1 & 2 & 0 & 1 & 4 \\
\hline & 3-4 grade & 0 & 0 & 1 & 0 & 0 & 1 \\
\hline \multirow[t]{2}{*}{ infection } & any grade & 1 & 0 & 2 & 0 & 1 & 4 \\
\hline & 3-4 grade & 0 & 0 & 1 & 0 & 0 & 1 \\
\hline $\begin{array}{l}\text { Febrile neutropenia } \\
\text { anemia }\end{array}$ & 3-4 grade & 0 & 0 & 2 & 0 & 0 & 2 \\
\hline \multirow[t]{2}{*}{ Vomit/nausea } & any grade & 1 & 3 & 2 & 1 & 2 & 9 \\
\hline & 3-4 grade & 0 & 0 & 0 & 0 & 1 & 1 \\
\hline \multirow[t]{2}{*}{ Abnormal liver function } & any grade & 1 & 1 & 1 & 0 & 1 & 4 \\
\hline & 3-4 grade & 0 & 0 & 0 & 0 & 0 & 0 \\
\hline Renal injury & any grade & 0 & 0 & 0 & 0 & 0 & 0 \\
\hline Fatigue & 3-4 grade & 0 & 0 & 1 & 0 & 1 & 2 \\
\hline Bleeding event & any grade & 0 & 0 & 0 & 0 & 0 & 0 \\
\hline Diarrhea & any grade & 0 & 0 & 0 & 0 & 0 & 0 \\
\hline Arrhythmia & any grade & 0 & 0 & 0 & 0 & 0 & 0 \\
\hline Diarrhea & any grade & 0 & 0 & 0 & 0 & 0 & 0 \\
\hline
\end{tabular}

CHOP, cyclophosphamide, epirubicin, vindesine, prednisone; DICE, dexamethasone, ifosfamide, carboplatin and etoposide; DHAP, dexamethasone, cytarabine and cis-platinum. $R$, rituximab; Zan, zanubrutinib.

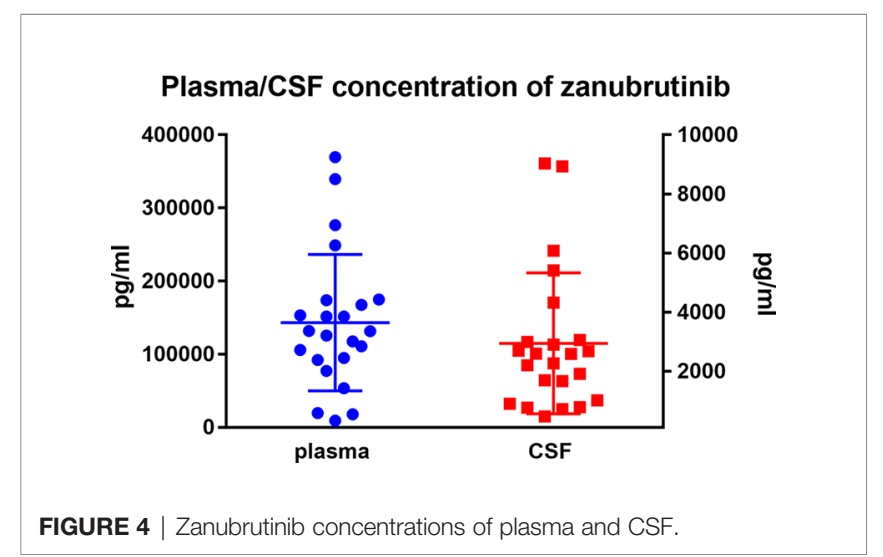

concentrations of zanubrutinib in $95.7 \%(22 / 23)$ of the samples were above the enzymatic IC50 values (ABC DLBCL lines in vitro, IC50 $=118 \mathrm{pg} / \mathrm{ml}, 0.4 \mathrm{nM}$; OCI-Ly-10, IC50 $=707.34 \mathrm{pg} /$ $\mathrm{ml} 1.5 \mathrm{nM})$.
A comparison of the zanubrutinib CSF/plasma ratios of different patients showed no significant effects of sex, DLBCL subtype, CNS involvement, or the co-administration of BBBpenetrating drugs $(P>0.1)$.

We compared the plasma and CSF concentrations of zanubrutinib at different time points $(2$ vs. $3 \mathrm{~h}$ after administration). While the plasma and CSF concentrations of zanubrutinib tended to be higher in the samples collected at $2 \mathrm{~h}$ after administration, the penetration ratios were similar between the two time points (Table 3 ).

Meanwhile, we tested reduplicated samples collected from six patients at the same time point but in different cycles. We observed huge variation in the CSF/plasma ratio among these patients, with values ranging from $10.5 \%$ to $78.1 \%$. However, the consistency was acceptable between cycles in the same patient (Figure 5 and Table S1).

We also tested the trough concentrations of zanubrutinib in two patients (case 1 and case 3) to evaluate if the CSF concentrations of zanubrutinib remained roughly above the IC50 value. The CSF

TABLE 3 | Zanubrutinib concentrations in plasma and CSF.

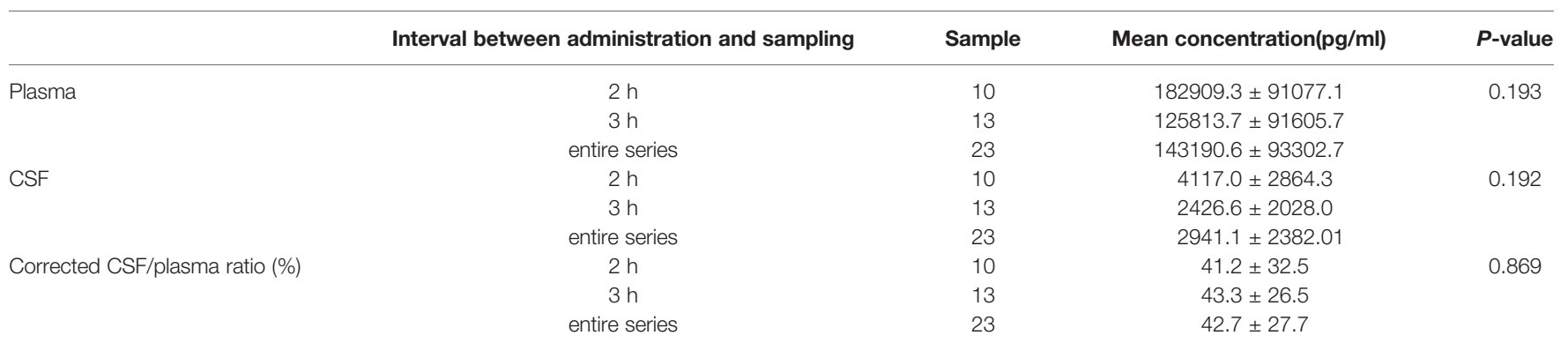


A

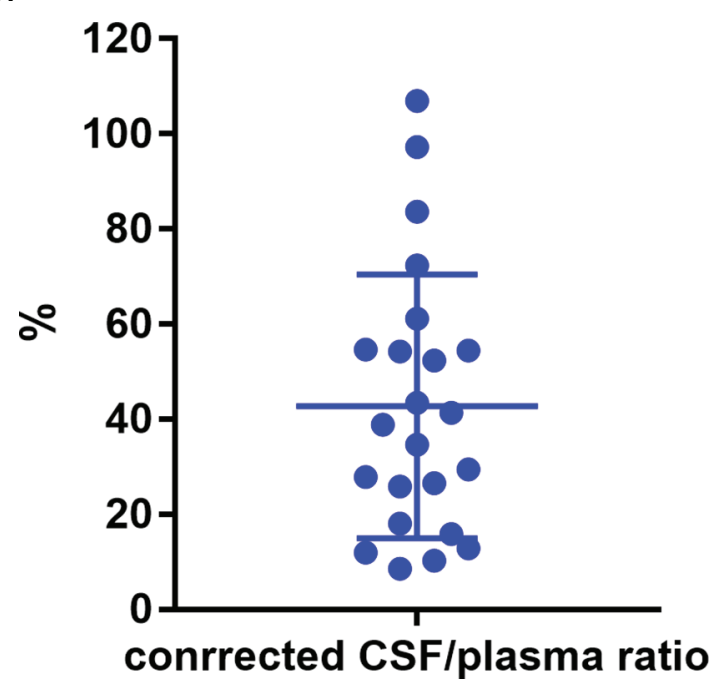

B

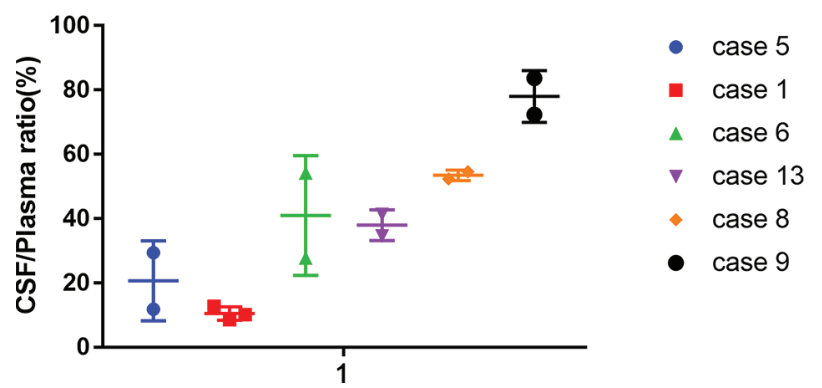

FIGURE 5 | Corrected CSF/plasma ratios for zanubrutinib. (A) 23 paired samples of Corrected CSF/plasma ratio by protein binding rate. (B) Corrected CSF/plasma ratios for zanubrutinib in different patients.

concentrations were 267 and $5022 \mathrm{pg} / \mathrm{ml}$ in case 1 and case 3, respectively, and case 3 showed a value below the IC50 value.

\section{DISCUSSION}

As mentioned above, there are great unmet needs in CNSL treatment and prophylaxis, BTK inhibitors (ibrutinib and tirabrutinib) show efficacy in CNSL. To the best of our knowledge, this is the first study to demonstrate the effectiveness of zanubrutinib-containing regimens in different subtypes of DLBCL, particularly CNS lymphoma. The high BBB penetration of zanubrutinib was confirmed by LC-MS/MS, which supported the use of zanubrutinib in CNSL.

This case study consisted of 13 DLBCL patients treated with zanubrutinib-containing regimens with an ORR of $84.6 \%$. Two relapsed cases with PCNSL received zanubrutinib alone as a leadin treatment at the beginning of salvage therapy, and both achieved significant disease control within $14 \mathrm{~d}$. Lionakis et a. reported a similar phenomenon for ibrutinib in 2017; they conducted a 14-d therapy with ibrutinib before applying TEDDI-R as a combination regimen, and $94 \%$ of patients showed disease improvement after the ibrutinib monotherapy (28). This promising result indicates that zanubrutinib can induce rapid remission in CNSL patients. Thus, zanubrutinib could be a good option for these patients.

We also evaluated the safety of zanubrutinib-containing regimens in DLBCL. The tolerability of zanubrutinib was favorable in this study. Significant toxicity was indicated by hematological events; grade 3-4 neutropenia and thrombocytopenia occurred in $54.5 \%$ and $11.1 \%$ of cases, respectively. All severe AEs occurred for intensive salvage regimens, including $\mathrm{HD}$ cytarabine, DICE, and DHAP. In a phase I study of ibrutinib combined with R-ICE for the treatment of transplant-eligible $\mathrm{r} / \mathrm{r}$ DLBCL, no dose-limiting toxicities were observed, even with ibrutinib at $840 \mathrm{mg}$ daily (29). In our study, both cases of relapsed DLBCL achieved CR with the zanubrutinib-containing regimens, and no unexpected serious AEs were observed. Meanwhile, no unique AEs related to BTK inhibitors (e.g., bleeding events, atrial fibrillation, and aspergillosis) were observed. The low incidence of BTK inhibitor-specific AEs was attributed to the limited number of cases in this study; the low off-target inhibitory activity of zanubrutinib may also have contributed (22).

However, zanubrutinib has not been approved in DLBCL/ PCNSL. We applied zanubrutinib in DLBCL/PCNSL based on the following scientific and ethical considerations. First, evidence regarding the efficacy of zanubrutinib in DLBCL emerged in 2017. At the 2017 American Society of Hematology meeting, Tam et al. reported that among 75 patients with non-Hodgkin lymphoma (including 23 with DLBCL) who were treated with zanubrutinib, 14 achieved remission (30). In 2021, Yang et al. reported the application of zanubrutinib in relapsed non-GCB (germinal center) DLBCL in a multicenter, single-arm, phase 2 study involving 41 patients (31). After a median follow-up time of 6.8 months, the ORR was $29.3 \%$, and the complete response rate was $17.1 \%$. AEs leading to treatment discontinuation were reported in four patients. Second, the efficacy and safety of zanubrutinib as a monotherapy or combined with R-CHOP were confirmed by data from two trials conducted at our center: Intravitreal MTX and ZR Regimen in Newly Diagnosed PVRL, NCT04899453 (Regimen 1); and Zanubrutinib Combined With R-CHOP in Newly-diagnosed Intravascular Large B-cell Lymphoma, NCT04899570 (Regimen 4). These results give us confidence to expand the application of zanubrutinib in DLBCL. Third, the best salvage regimen was undermined in relapsed DLBCL and PCNSL, and clinical trials were preferred. The patients from Regimen 2 and Regimen 3 were relapsed/ refractory $(r / r)$ DLBCL with/without CNS involved; these patients were treated with a zanubrutinib-containing regimen after fully understanding the benefits and risks and signing the informed consent forms. All activities were approved by the Institutional Review Board of our institution.

In this study, we analyzed the distribution of zanubrutinib in CSF for the first time. Some issues should be clarified. First, zanubrutinib maybe a suitable candidate for primary and 
TABLE 4 | Plasma and CSF concentrations of different BTK inhibitors.

\begin{tabular}{|c|c|c|c|c|c|c|}
\hline \multirow{4}{*}{$\begin{array}{l}\text { Dose }(\mathrm{mg}) \\
\text { Mean Plasma }(\mathrm{ng} / \mathrm{ml}) \\
\text { Mean CSF }(\mathrm{ng} / \mathrm{ml})\end{array}$} & \multirow{2}{*}{\multicolumn{2}{|c|}{$\begin{array}{l}\text { Ibrutinib } \\
\text { 700mg qd }\end{array}$}} & \multirow{3}{*}{$\begin{array}{c}840 \mathrm{mg} \text { qd } \\
875.6\end{array}$} & \multicolumn{2}{|c|}{ Tirabrutinib } & \multirow{2}{*}{$\frac{\text { Zanubrutinib }}{160 \mathrm{mg} \text { bid }}$} \\
\hline & & & & $320 \mathrm{mg}$ qd & 480mg qd & \\
\hline & 53.7 & 217.4 & & 16.3 & 77.0 & 143.2 \\
\hline & 0.62 & 0.87 & 0.59 & 2.19 & 14.0 & 2.94 \\
\hline
\end{tabular}

secondary CNSL treatment with outstanding BBB permeability. The median CSF/plasma ratio of zanubrutinib was around $2 \%$; after adjusting for the amount of protein-bound drug, the CSF/ plasma ratio increased to nearly $40 \%$. This significant change demonstrates that zanubrutinib penetrated the $\mathrm{BBB}$ in its free form. Several studies have demonstrated the high brain distribution level of ibrutinib in animal models and human biological specimens $(15,16,18)$. In 2016, Soussain et al. confirmed that ibrutinib can penetrate in the BBB, with CSF concentrations above the IC50 level consistent with clinical response (14). In 2017, Lionakis et al. confirmed the high efficacy of an ibrutinib-containing regimen (TEDDi-R) in CNS lymphomas and evaluated the pharmacokinetics of ibrutinib in plasma and CSF samples (28). After adjusting for a protein binding of $97.3 \%$, the authors found a CSF/plasma ratio of $28.7 \%$ (23.2\%-446.6\%), and the median time that the CSF concentration remained above the enzymatic IC50 level was $4 \mathrm{~h}(0-24 \mathrm{~h})$ at a dose of $840 \mathrm{mg}$. In 2018, Goldwirt et al. developed a Swiss mouse model to evaluate the distribution of ibrutinib in the brain and found that ibrutinib crossed the BBB and was highly distributed in the brain tissue (18). In 2020, Narita et al. reported the CSF concentration of tirabrutinib in PCNSL patients, the trough concentrations at $320 \mathrm{mg}$ and $480 \mathrm{mg}$ were $2.19 \pm 0.476$ and $14.0 \pm 8.92) \mathrm{ng} / \mathrm{mL}$, respectively (19). We compared the plasma and CSF concentrations of different BTK inbihotors (see Table 4), zanubrutinib had higher CSF concentration than ibrutinib and low dose tirabrutinib.

Second, according to the package insert, the median time to maximum concentration of zanubrutinib is $2 \mathrm{~h}$, resulting in a mean half-life of approximately 2-4 h. We then compared the CSF and plasma concentrations of zanubrutinib at different time points. The plasma concentrations of zanubrutinib were higher at $2 \mathrm{~h}$ after administration compared to at $4 \mathrm{~h}$ after administration; a similar trend was found in the CSF concentrations. This suggests similar dynamics in the CSF and plasma concentrations of zanubrutinib, with rapid and efficient $\mathrm{BBB}$ crossing. This distribution behavior of zanubrutinib is similar to that of ibrutinib (18).

Another issue is the absolute zanubrutinib concentration in the CSF. The difference between the highest and lowest zanubrutinib concentrations was almost 200 times, with values ranging from 466 to $8930 \mathrm{pg} / \mathrm{ml}$. Zanubrutinib showed antitumor activity in the pg range in two ABC DLBCL lines $($ TMD8, IC50 = $118 \mathrm{pg} / \mathrm{ml}$; OCI-Ly-10, IC50 = $707.34 \mathrm{pg} / \mathrm{ml})$ (32). While a zanubrutinib concentration below the IC50 value was found in one CSF sample, the mean concentration of zanubrutinib in the CSF was much higher than the IC50 value.
The lowest concentration was detected in case 9, a female patient who showed a rapid response to zanubrutinib monotherapy. This highlighted the potential effect of zanubrutinib/ibrutinib for prevention of CNS relapse in DLBCL.

This preliminary study had several limitations. First, the present results are based on a limited number of patients, and the DLBLC and treatment subtypes were heterogeneous. This may generate selection bias. Second, detailed pharmacokinetic data were not collected for zanubrutinib. No Ommaya reservoirs were placed in the patients, and CSF samples were collected by lumbar puncture, allowing for only one sample during each 24-h treatment cycle. Without dynamic monitoring, comprehensive information about the CSF distribution, including how long the CSF concentration stayed above the IC50 value and the area under the curve of CSF, could not be obtained. Finally, prolonged effectiveness was not evaluated because of the limited observation period.

\section{CONCLUSIONS}

The high response rate and good tolerance observed in this study suggest that zanubrutinib-containing regimens are a promising treatment option for DLBCL, especially CNSL. Zanubrutinib could effectively cross the $\mathrm{BBB}$. The results provide preliminarily evidence for the application of zanubrutinib in CNS lymphoma.

\section{DATA AVAILABILITY STATEMENT}

The original data presented in this paper are included in the article/Supplementary Material. Further inquiries can be directed to the corresponding author.

\section{ETHICS STATEMENT}

The studies involving human participants were reviewed and approved by Institutional Review Board of Peking Union Medical College Hospital. The patients/participants provided their written informed consent to participate in this study.

\section{AUTHOR CONTRIBUTIONS}

$\mathrm{YZ}, \mathrm{YL}$, and $\mathrm{ZZ}$ performed the research. $\mathrm{YZ}$ and $\mathrm{WZ}$ designed the study. YZ, WW, DQZ, and CW took care of the patients. YZ 
analyzed the data. YZ wrote the paper. DBZ supervised the study and revised the paper. All authors read and revised the manuscript, and all authors have approved the submitted version.

\section{FUNDING}

This study was funded by the CAMS Innovation Fund for Medical Sciences (CIFMS) [2021-I2M-C\&T-B-005] and CAMS Innovation Fund for Medical Sciences (CIFMS) [2019I2M2-009].

\section{REFERENCES}

1. Rickert RC. New Insights Into Pre-BCR and BCR Signalling With Relevance to B Cell Malignancies. Nat Rev Immunol (2013) 13:578-91. doi: 10.1038/ nri3487

2. Wilson WH, Young RM, Schmitz R, Yang Y, Pittaluga S, Wright G, et al. Targeting B Cell Receptor Signaling With Ibrutinib in Diffuse Large B Cell Lymphoma. Nat Med (2015) 21:922-6. doi: 10.1038/nm.3884

3. Wilson WH, Wright GW, Huang DW, Hodkinson B, Balasubramanian S, Fan Y, et al. Effect of Ibrutinib With R-CHOP Chemotherapy in Genetic Subtypes of DLBCL. Cancer Cell (2021) 39(12):1643-53. doi: 10.1016/ j.ccell.2021.10.006

4. Grommes C, DeAngelis LM. Primary CNS Lymphoma. J Clin Oncol (2017) 35:2410-8. doi: 10.1200/JCO.2017.72.7602

5. Holdhoff M, Mrugala MM, Grommes C, Kaley TJ, Swinnen LJ, PerezHeydrich C, et al. Challenges in the Treatment of Newly Diagnosed and Recurrent Primary Central Nervous System Lymphoma. JNCCN J Natl Compr Cancer Netw (2020) 18:1571-8. doi: 10.6004/JNCCN.2020.7667

6. Nau R, Sörgel F, Eiffert H. Penetration of Drugs Through the BloodCerebrospinal Fluid/Blood-Brain Barrier for Treatment of Central Nervous System Infections. Clin Microbiol Rev (2010) 23:858-83. doi: 10.1128/ CMR.00007-10

7. Ferreri AJM, Cwynarski K, Pulczynski E, Ponzoni M, Deckert M, Politi LS, et al. Chemoimmunotherapy With Methotrexate, Cytarabine, Thiotepa, and Rituximab (MATRix Regimen) in Patients With Primary CNS Lymphoma: Results of the First Randomisation of the International Extranodal Lymphoma Study Group-32 (IELSG32) Phase 2 Trial. Lancet Haematol (2016) 3:e217-27. doi: 10.1016/S2352-3026(16)00036-3

8. Morris PG, Correa DD, Yahalom J, Raizer JJ, Schiff D, Grant B, et al. Rituximab, Methotrexate, Procarbazine, and Vincristine Followed by Consolidation Reduced-Dose Whole-Brain Radiotherapy and Cytarabine in Newly Diagnosed Primary CNS Lymphoma: Final Results and Long-Term Outcome. J Clin Oncol (2013) 31:3971-9. doi: 10.1200/JCO.2013.50.4910

9. Puckrin R, El Darsa H, Ghosh S, Peters A, Owen C, Stewart D. Ineffectiveness of High-Dose Methotrexate for Prevention of CNS Relapse in Diffuse Large B Cell Lymphoma. Am J Hematol (2021) 96(7):764-71. doi: 10.1002/ajh.26181

10. Eyre TA, Djebbari F, Kirkwood AA, Collins GP. Efficacy of Central Nervous System Prophylaxis With Stand-Alone Intrathecal Chemotherapy in Diffuse Large B-Cell Lymphoma Patients Treated With Anthracycline-Based Chemotherapy in the Rituximab Era: A Systematic Review. Haematologica (2020) 105:1914-24. doi: 10.3324/haematol.2019.229948

11. Bernard S, Goldwirt L, Amorim S, Brice P, Brière J, De Kerviler E, et al. Activity of Ibrutinib in Mantle Cell Lymphoma Patients With Central Nervous System Relapse. Blood (2015) 126:1695-8. doi: 10.1182/blood2015-05-647834

12. Chamoun K, Choquet S, Boyle E, Houillier C, Larrieu-Ciron D, Al Jijakli A, et al. Ibrutinib Monotherapy in Relapsed/Refractory CNS Lymphoma: A Retrospective Case Series. Neurology (2017) 88:101-2. doi: 10.1212/ WNL.0000000000003420

13. Soussain C, Choquet S, Blonski M, Leclercq D, Houillier C, Rezai K, et al. Ibrutinib Monotherapy for Relapse or Refractory Primary CNS Lymphoma and Primary Vitreoretinal Lymphoma: Final Analysis of the Phase II 'Proofof-Concept' iLOC Study by the Lymphoma Study Association (LYSA) and the

\section{ACKNOWLEDGMENTS}

The authors thank AiMi Academic Services (www.aimieditor. com) for the English language editing and review services.

\section{SUPPLEMENTARY MATERIAL}

The Supplementary Material for this article can be found online at: https://www.frontiersin.org/articles/10.3389/fonc.2021. 760405/full\#supplementary-material

French Oculo-Cerebral Lymphoma (LOC) Net. Eur J Cancer (2019) 117:12130. doi: 10.1016/j.ejca.2019.05.024

14. Cabannes-Hamy A, Lemal R, Goldwirt L, Poulain S, Amorim S, Pérignon R, et al. Efficacy of Ibrutinib in the Treatment of Bing-Neel Syndrome. Am J Hematol (2016) 91:E17-9. doi: 10.1002/ajh.24279

15. Beauvais D, Goossens J-F, Boyle E, Allal B, Lafont T, Chatelut E, et al. Development and Validation of an UHPLC-MS/MS Method for Simultaneous Quantification of Ibrutinib and Its Dihydrodiol-Metabolite in Human Cerebrospinal Fluid. J Chromatogr B (2018) 1093-1094:158-66. doi: 10.1016/j.jchromb.2018.06.026

16. Yasu T, Momo K, Yasui H, Kuroda S. Simple Determination of Plasma Ibrutinib Concentration Using High-Performance Liquid Chromatography. BioMed Chromatogr (2019) 33:1-6. doi: 10.1002/bmc.4435

17. Sun S, Cheng D, Kong S, Li X, Li T, Yu Q, et al. A Rapid and Sensitive Method for Quantification of Ibrutinib in Rat Plasma by UPLC-ESI-MS/MS: Validation and Application to Pharmacokinetic Studies of a Novel Ibrutinib Nanocrystalline. BioMed Chromatogr (2020) 34:1-8. doi: 10.1002/bmc.4703

18. Goldwirt L, Beccaria K, Ple A, Sauvageon H, Mourah S. Ibrutinib Brain Distribution: A Preclinical Study. Cancer Chemother Pharmacol (2018) 81:783-9. doi: 10.1007/s00280-018-3546-3

19. Narita Y, Nagane M, Mishima K, Terui Y, Arakawa Y, Yonezawa H, et al. Phase I/II Study of Tirabrutinib, a Second-Generation Bruton's Tyrosine Kinase Inhibitor, in Relapsed/Refractory Primary Central Nervous System Lymphoma. Neuro Oncol (2021) 23:122-33. doi: 10.1093/neuonc/noaa145

20. Xu W, Yang S, Zhou K, Pan L, Li Z, Zhou J, et al. Treatment of Relapsed/ Refractory Chronic Lymphocytic Leukemia/Small Lymphocytic Lymphoma With the BTK Inhibitor Zanubrutinib: Phase 2, Single-Arm, Multicenter Study. J Hematol Oncol (2020) 13:48. doi: 10.1186/s13045-020-00884-4

21. Song Y, Zhou K, Zou D, Zhou J, Hu J, Yang H, et al. Treatment of Patients With Relapsed or Refractory Mantle-Cell Lymphoma With Zanubrutinib, a Selective Inhibitor of Bruton's Tyrosine Kinase. Clin Cancer Res (2020) 26:4216-24. doi: 10.1158/1078-0432.CCR-19-3703

22. Tam CS, Opat S, D'Sa S, Jurczak W, Lee HP, Cull G, et al. A Randomized Phase 3 Trial of Zanubrutinib vs Ibrutinib in Symptomatic Waldenström Macroglobulinemia: The ASPEN Study. Blood (2020) 136:2038-50. doi: 10.1182/BLOOD.2020006844

23. Guo Y, Liu Y, Hu N, Yu D, Zhou C, Shi G, et al. Discovery of Zanubrutinib (BGB-3111), A Novel, Potent, and Selective Covalent Inhibitor of Bruton's Tyrosine Kinase. J Med Chem (2019) 62:7923-40. doi: 10.1021/ acs.jmedchem.9b00687

24. Abrey LE, Batchelor TT, Ferreri AJM, Gospodarowicz M, Pulczynski EJ, Zucca E, et al. Report of an International Workshop to Standardize Baseline Evaluation and Response Criteria for Primary CNS Lymphoma. J Clin Oncol (2005) 23:5034-43. doi: 10.1200/JCO.2005.13.524

25. Cheson BD, Fisher RI, Barrington SF, Cavalli F, Schwartz LH, Zucca E, et al. Recommendations for Initial Evaluation, Staging, and Response Assessment of Hodgkin and Non-Hodgkin Lymphoma: The Lugano Classification. J Clin Oncol (2014) 32:3059-67. doi: 10.1200/JCO.2013.54.8800

26. Nguyen-Them L, Costopoulos M, Tanguy ML, Houillier C, Choquet S, Benanni $\mathrm{H}$, et al. The CSF IL-10 Concentration Is an Effective Diagnostic Marker in Immunocompetent Primary CNS Lymphoma and a Potential Prognostic Biomarker in Treatment-Responsive Patients. Eur J Cancer (2016) 61:69-76. doi: 10.1016/j.ejca.2016.03.080 
27. Zhang Y, Zou D, Yin J, Zhang L, Zhang X, Wang W, et al. Changes in Cerebrospinal Fluid Interleukin-10 Levels Display Better Performance in Predicting Disease Relapse Than Conventional Magnetic Resonance Imaging in Primary Central Nervous System Lymphoma. BMC Cancer (2021) 21:183. doi: 10.1186/s12885-020-07774-5

28. Lionakis MS, Dunleavy K, Roschewski M, Widemann BC, Butman JA, Schmitz $\mathrm{R}$, et al. Inhibition of B Cell Receptor Signaling by Ibrutinib in Primary CNS Lymphoma. Cancer Cell (2017) 31:833-43.e5. doi: 10.1016/j.ccell.2017.04.012

29. Sauter CS, Matasar MJ, Schoder H, Devlin SM, Drullinsky P, Gerecitano J, et al. A Phase 1 Study of Ibrutinib in Combination With R-ICE in Patients With Relapsed or Primary Refractory DLBCL. Blood (2018) 131:1805-8. doi: 10.1182/blood-2017-08-802561

30. Tam CS, Simpson D, Opat S, Kim WS, Wang M CG. Safety and Activity of the Highly Specific BTK Inhibitor BGB-3111 in Patients With Indolent and Aggressive Non Hodgkin's Lymphoma. Blood (2017) 130:152. doi: 10.1182/blood.V130

31. Yang H, Xiang B, Song Y, Zhang H, Zhao W, Zou D, et al. Zanubrutinib Monotherapy for Relapsed or Refractory Non-Germinal Center Diffuse Large B-Cell Lymphoma. Blood Adv (2021) 12:bloodadvances.2020003698. doi: 10.1182/bloodadvances.2020003698

32. Tarantelli C, Zhang L, Curti E, Gaudio E, Spriano F, Priebe V, et al. The Bruton Tyrosine Kinase Inhibitor Zanubrutinib (BGB-3111) Demonstrated
Synergies With Other Anti-Lymphoma Targeted Agents. Haematologica (2019) 104:e307-9. doi: 10.3324/haematol.2018.214759

Conflict of Interest: The authors declare that the research was conducted in the absence of any commercial or financial relationships that could be construed as a potential conflict of interest.

Publisher's Note: All claims expressed in this article are solely those of the authors and do not necessarily represent those of their affiliated organizations, or those of the publisher, the editors and the reviewers. Any product that may be evaluated in this article, or claim that may be made by its manufacturer, is not guaranteed or endorsed by the publisher.

Copyright (c) 2021 Zhang, Li, Zhuang, Wang, Wei, Zhao, Zhou and Zhang. This is an open-access article distributed under the terms of the Creative Commons Attribution License (CC BY). The use, distribution or reproduction in other forums is permitted, provided the original author(s) and the copyright owner(s) are credited and that the original publication in this journal is cited, in accordance with accepted academic practice. No use, distribution or reproduction is permitted which does not comply with these terms. 